\title{
Shape optimization by the level-set method applied to architectured flexural panels
}

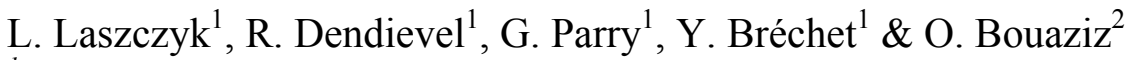 \\ ${ }^{I}$ SIMAP, Grenoble Institute of Technology, CNRS, UJF, France \\ ${ }^{2}$ ArcelorMittal Research, France
}

\begin{abstract}
Architectured materials (such as foams, corrugated structures, trusses, hollow spheres) are used, alone or as core material in a sandwich structure, for flexural panels. The idea is to take advantage of the architecture to combine multiple functionalities (e.g. lightweight, stiffness, thermal insulation). Therefore it seems relevant to design the distribution of matter in order to obtain the desired performances. Multi-functional and conflicting specifications lead to non-trivial selection and/or optimization problems. It is hence proposed to use numerical shape optimization procedures into a "material by design" approach applied to periodically architectured flexural panels.

A finite element analysis on the unit cell is presented to compute the effective stiffness of each panel depending on its architecture. Then, shape optimization by the level-set method is made on the unit cell with regard to the effective stiffness and a volume constraint. Multiple start geometries and objective functions are tested. The four-point bending test, which usually characterizes flexural panels performance, is used as a reference for both homogenization and optimization.
\end{abstract}

Keywords: shape optimization, level-set method, homogenization, flexural panel, sandwich structures, architectured materials.

\section{Introduction}

Specifications in automotive industries are more and more complex in the way that multi-functional performances (such as stiffness, thermal and acoustic insulation) are required while keeping the weight as low as possible. Composite and architectured materials often give efficient solutions. Recently, numerous 
techniques have been developed for manufacturing lightweight structures like graded metal foams 0 , hollow spheres 0 and trusses 0 , corrugated structures, honeycombs and other cellular solids 0 . In a "material by design" approach 0 , finding the cellular architecture that combines in the most efficient way a given set of conflicting properties is the key issue. In this regard, shape optimization techniques can enlarge the degrees of freedom on the geometry.

The proposed example of using shape optimization techniques to design a cellular material is the following. It consists of optimizing the matter distribution of a periodically architectured panel to obtain the highest stiffness with respect to a given relative density. The stiffness of such flexural panels is usually characterized by the four-point bending stiffness that includes flexion and shear contributions. Therefore, the proposed optimization problem is multi-functional and deals with maximizing the flexural and the shear stiffness with a volume constraint. Since the optimized panel is wished periodic, the optimization domain is the unit cell and the periodicity could be seen as an additional constraint.

The considered architectured panels (fig. 1 and 2) are assumed to be periodic in the $\mathrm{x}$-direction and invariant in the y-direction. Only the bending stiffness in the $\mathrm{x}$-direction is studied.

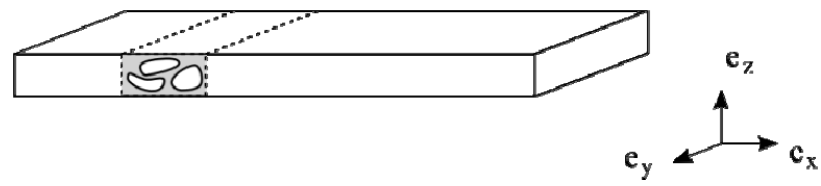

Figure 1: Generic periodically architectured panel. The xy-plane is the inplane of the panel. The xz-plane is the one of the $2 \mathrm{D}$ plain strain model since the geometry is invariant in the y-direction.

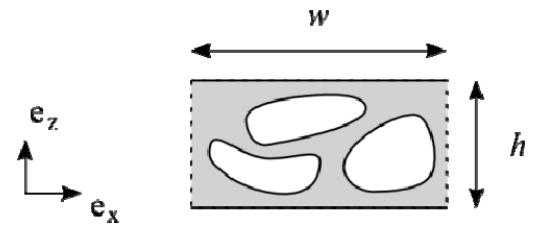

Figure 2: $\quad$ Unit cell of a generic periodically architectured panel.

The two following sections present, respectively, the homogenization procedure used to estimate the performance of each geometry, and the optimization results that use the previous procedure at each step of the algorithm.

First, in Section 2, an homogenization method is described. It allows one to estimate the stiffness of any architectured panel, computing on the unit cell the effective flexion and shear contributions commonly used for flexural panels. This method is then validated comparing the four-point bending stiffness of few test architectures, on one side analytically calculated with effective stiffness 
coefficients and, on the other side, numerically computed with a whole panel finite element simulation.

Finally, in Section 3, shape optimization by the level-set method is made on the unit cell to maximize the effective coefficients. Since this problem is multiobjective, the choice of the objective function is central, then different functions such as linear combination, product of power or physically based function are discussed.

\section{Homogenization}

The stiffness of such considered panels is usually characterized by the four-point bending stiffness, which has an analytical expression in the homogeneous case function of the flexural and shear coefficients. These stiffness coefficients include both material properties, such as Young's and shear modulus, and geometrical dimensions, such as moment of inertia and thickness.

In the proposed homogenization procedure, effective coefficients are numerically computed by an energy approach, so that the bending stiffness of the whole architectured panels can be estimated by the one of the effective homogeneous panel.

\subsection{Homogeneous panel}

The mechanical behaviour of a panel under four-point bending load, fig. 3, is usually characterized (see Ashby et al. [6]) by the bending stiffness $R_{f}$ defined as:

$$
F=R_{f} d
$$

where $F$ is the distributed applied force and $d$ is the deflexion of the inner indenters. The distance between inner and outer indenters are denoted respectively $s$ and $l$.

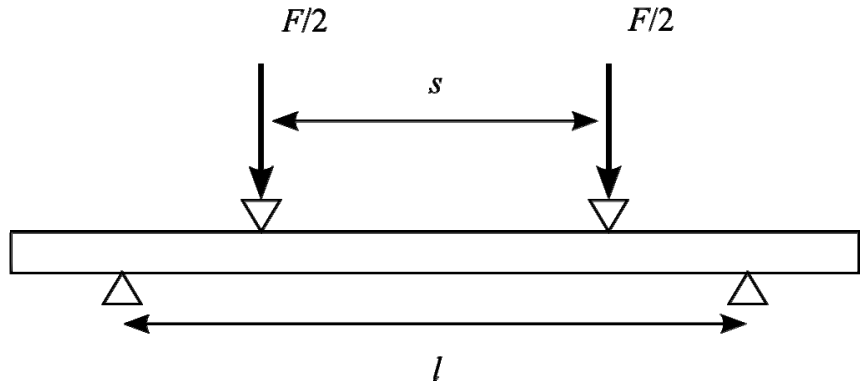

Figure 3: Four-point bending test.

Considering the flexural behaviour in the x-direction, Allen [7] gives an analytical expression of this bending stiffness for an homogeneous panel as: 


$$
\frac{1}{R_{f}}=\frac{(l-s)^{2}(l+2 s)}{48 D_{x}}+\frac{l-s}{4 D_{x z}}
$$

where $D_{x}$ is the flexural coefficient given by:

$$
D_{x}=\frac{E}{1-v^{2}} \frac{h^{3}}{12}
$$

and $D_{x z}$ is the shear coefficient given by:

$$
D_{x z}=G h
$$

The analytical expression of this bending stiffness can be compared to numerical results. With taking the invariance of the mechanical problem in the $y$ direction into account, a 2D plain strain finite element simulation is done on an homogeneous panel, fig. 3. A loading force $F$ is applied and the strain energy $W^{*}$ is computed. Then, the stiffness is obtained through the following equation:

$$
\frac{1}{R_{f}}=\frac{2 W^{*}}{F^{2}}
$$

Figure 5 shows the agreement between the analytical model and the finite element simulation of the whole homogeneous panel (resp. black line and black circles).

\subsection{Effective stiffness coefficients calculations}

Let us present the way to compute the effective stiffness coefficients of any periodically architectured panel. Each coefficient, respectively for flexion and shear, is computed by a finite element simulation of the unit cell under the corresponding elementary loading.

\subsubsection{Flexural stiffness coefficient}

To compute the flexural coefficient, the unit cell is submitted to an average curvature $C$ and mixed boundary conditions (Neumann in the y-direction and periodic in the $\mathrm{x}$-direction), see Buannic et al. [8]. The analytical corresponding strain field for the homogeneous unit cell is:

$$
\hat{\varepsilon}=C y\left(\mathrm{e}_{\mathrm{x}} \otimes \mathrm{e}_{\mathrm{x}}-\frac{v}{1-v} \mathrm{e}_{\mathrm{y}} \otimes \mathrm{e}_{\mathrm{y}}\right)
$$

where $\otimes$ denotes the dyadic product.

The present finite element simulation computes the displacements $u^{\#}$ which correspond to a fluctuation strain field around $\hat{\varepsilon}$. That is to say that the mechanical problem is solved with a total strain tensor equal to: 


$$
\varepsilon=\hat{\varepsilon}+\varepsilon\left(u^{\#}\right)
$$

Then, the effective flexural coefficient is obtained by equalling the resulting strain energy $W^{*}$ to its analytic expression in the homogeneous case. It leads to:

$$
\widetilde{D}_{x}=\frac{2 W^{*}}{w C^{2}}
$$

\subsubsection{Shear stiffness coefficient}

To compute the shear coefficient, prescribed displacement boundary conditions are applied to the upper and lower faces $\left(u_{x}=\delta\right.$ on the upper face, and $u_{x}=0$ on the lower one).

As previously, the effective shear coefficient is obtained by equalling the computed strain energy $W^{*}$ to its analytic expression in the homogeneous case. It leads to:

$$
\widetilde{D}_{x z}=\frac{2 W^{*} h^{2}}{w \delta^{2}}
$$

\subsection{Comparison between homogenized and architectured panels for few examples}

Before optimizing the geometry with regard to these effective stiffness coefficients, it seems interesting to compare the four-point bending stiffness estimated with Allen's expression, eqn. (2), substituting the coefficients by the effective one, and computed with a finite element simulation on the whole architectured panel.

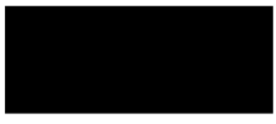

Homogeneous

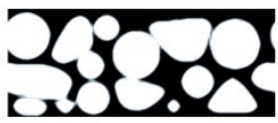

Random

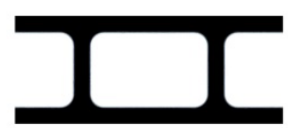

Square

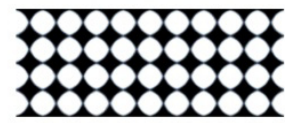

Cubic

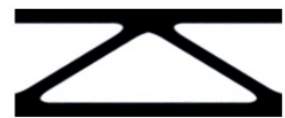

Triangle

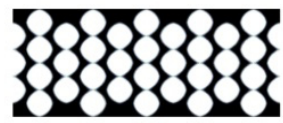

Cubic centred

Figure 5: $\quad$ Sketch of the different tested designs, fig. 4. The relative density is 0,4 for all except the homogeneous one. 
It is obvious that the kinematic assumptions used by Allen that leads to eqn. (2) are no more valid when the panel is architectured. But let us even do it and evaluate how far this estimation is from the finite element computations results. Figure 5 compares the four-point bending stiffness estimated via eqn. (2) with the one computed by finite element method similarly to Section 2.1, for different designs shown in fig. 5 . The relative density is 0,4 for all the designs except the homogeneous one.
- Allen homogeneous
- FEM homogeneous
--- Allen square
口 FEM square
- - - Allen triangle
$\triangle \quad$ FEM triangle
-.- - Allen random
$\times \quad$ FEM random
------- Allen simple cubic
- FEM simple cubic
Allen centered cubic
c) FEM centered cubic

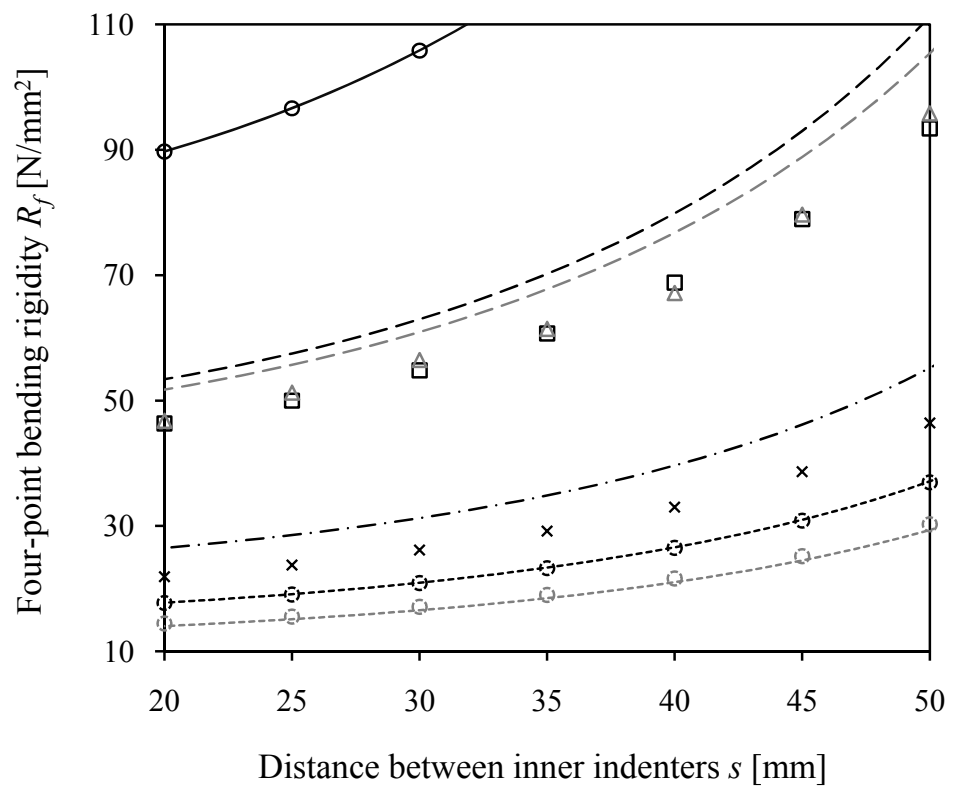

Figure 5: Comparison between the four-point bending stiffness estimated with the effective coefficients and computed by the finite element method, for different designs.

It can be observed that the Allen analytical model used with the effective coefficients identified on a periodic unit seems still to be correct for refined architectures (i.e. architectures having a characteristic length small enough compared to the plate thickness). For highly heterogeneous architectures, the model appears stiffer but still gives a good order of magnitude. Two main reasons could explain this shift. First, the kinematic of deformation is strongly 
modified in heterogeneous architectures so that the analytic expression is not valid anymore, as was said above. For example, small fluctuations of the computed stiffness around a smooth evolution for the triangle design are observed, which is due to the position of the inner indenters relatively to the internal diagonal beams. The second reason is that the computation of the shear coefficient is made with prescribed displacement boundary conditions, well known to be leading to a stiffer effective behaviour.

\section{Shape optimization}

For any design of architectured panel, the previous homogenization method enables one to compute the corresponding flexural and shear coefficients appearing in eqn. (2) and to estimate the four-point bending stiffness. Using these coefficients as variables of a multi-objective function $J\left(D_{x}, D_{x z}\right)$, shape optimization can be performed to give an idea of the design that could give the best performance for a cellular flexural panel with a given relative density.

\subsection{Algorithm}

In order to enable topology changes and to avoid virtual composite matter, the shape representation is done by the level-set method (Eschenauer and Olhoff [9]). All admissible shapes $\Omega$ are included in the working domain $D$, which is the unit cell in this case. Then, the shape is captured by the level-set function defined as:

$$
\left\{\begin{array}{l}
\psi(x)=0 \Leftrightarrow \partial \Omega \cap D \\
\psi(x)<0 \Leftrightarrow \Omega \\
\psi(x)>0 \Leftrightarrow D \backslash \bar{\Omega}
\end{array}\right.
$$

The algorithm modifies the geometry at each iteration transporting the level-set function according to the Hamilton-Jacobi equation:

$$
\frac{\partial \psi}{\partial t}+V|\nabla \psi|=0
$$

where $V$ is the velocity chosen with regard to the shape derivative.

The rigorous definition of the shape derivative as well as its analytical expressions for the compliance of the linearized elasticity problem are given by Allaire et al. [10]. Let us just remind ourselves that for the strain energy, which expression is:

$$
W^{*}(\Omega)=\frac{1}{2} \int_{\Omega} A \varepsilon(u): \varepsilon(u) d x,
$$

the shape derivative in the $\theta$-direction, due to Cea et al. [11], is given by: 


$$
\frac{\partial W^{*}}{\partial \Omega}(\Omega)(\theta)=-\frac{1}{2} \int_{\partial \Omega} A \varepsilon(u): \varepsilon(u) \theta \cdot n d s
$$

where $u$ is the solution of the elasticity problem (self-adjoint) and $n$ the normal vector to the boundary.

Since the previously defined effective stiffness coefficients $\widetilde{D}_{x}$ and $\widetilde{D}_{x z}$ are proportional to the strain energy, the shape derivative of the following multiobjective functions will be derived from eqn. (13).

Finally, it can be demonstrated (see Allaire et al. [10]) that choosing adequately the velocity $V$ equal to the integrand of the shape derivative leads to a transformation of the shape in a direction that increases the objective function. Therefore, for each iteration, finite element simulations on a regular grid (Q2 elements) are performed to compute the effective coefficients and their shape derivative. Then, a resolution of the transport equation (11) with an explicit first order upwind scheme (see Sethian [12]) modifies the design of the architectured unit cell. As detailed by Allaire et al. [10], the level-set function is periodically reconditioned to the signed function to ensure sufficient regularity.

\subsection{Shape optimization on the unit cell}

This shape optimization algorithm was used to optimize the distribution of matter in the unit cell initialized with the random or the centered cubic designs (fig. 4). The two objectives to be maximized are the effective flexural and shear coefficients, with a constraint on the relative density that have been chosen equal to 0,4 . The results for two objective functions are presented: the weighted sum (as proposed by Ashby [13]) and the weighted product of the normalized stiffness coefficients.

\subsubsection{Objective function as a weighted sum}

The following results are obtained with an objective function as a weighted sum of the normalized effective coefficients:

$$
J_{1}=\alpha \frac{\widetilde{D}_{x}}{D_{x}}+(1-\alpha) \frac{\widetilde{D}_{x z}}{D_{x z}}
$$

where $\widetilde{D}_{i}$ are the effective coefficients, eqns. (8) and (9), and $D_{i}$ are the homogeneous coefficients, eqns. (3) and (4).

Figures 6 and 7 show the architecture evolution of the unit cell during optimization with regard to the shear coefficient only $(\alpha=0)$, whereas figures 8 and 9 shows the one with regard to the flexural coefficient only $(\alpha=1)$.

The computed optimized shapes confirm the engineer intuition. The optimization of the shear coefficient promotes diagonal beams, whereas the one of the flexural coefficient displace the matter as far as possible of the neutral axis of bending. 


\section{$8 \%$}
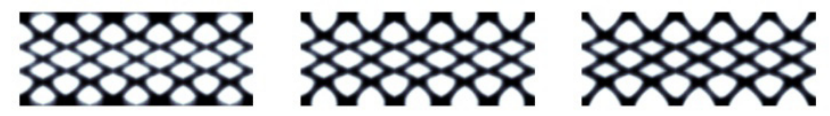

Figure 6: Iterations $0,10,20$ and 30 of the unit cell initialized with the centered cubic architecture and with $\alpha=0$.
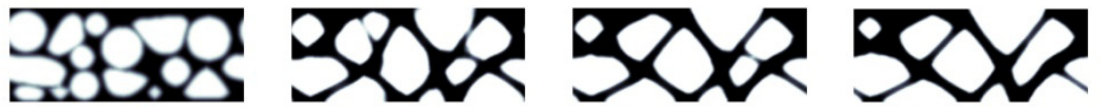

Figure 7: Iterations $0,10,20$ and 30 of the unit cell initialized with the random architecture and with $\alpha=0$.
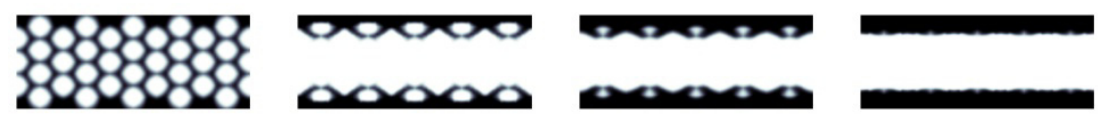

Figure 8: Iterations $0,5,10$ and 15 of the unit cell initialized with the centered cubic architecture and with $\alpha=1$.
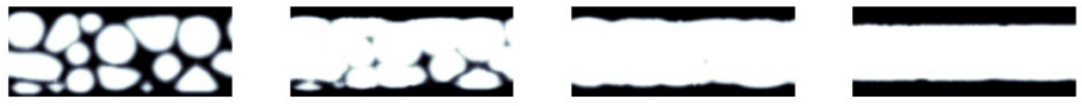

Figure 9: Iterations $0,5,10$ and 15 of the unit cell initialized with the random architecture and with $\alpha=1$.

For intermediate values of the weight $\alpha$, the optimized shapes are strictly identical to the one obtained with $\alpha=0$ or 1 . It seems that one of the coefficient is quickly neglected in order to only promote the other. The decrease of the neglected coefficient appears slower than the increase of the promoted one, so that the total objective function increases.

\subsubsection{Objective function as a weighted product}

The following results are obtained with an objective function as a weighted product of the effective coefficients:

$$
J_{2}=\left(\frac{\widetilde{D}_{x}}{D_{x}}\right)^{\beta}\left(\frac{\widetilde{D}_{x z}}{D_{x z}}\right)^{1-\beta}
$$

Figures 10 and 11 compare the optimized architectures obtained after 30 iterations with different values of the weight $\beta$. Gradually from $\beta=0,2$ to 0,8 , the observed tendency is to grow the upper and lower faces as well as to reduce the number and the size of diagonal beams. 


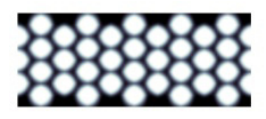

Initial shape

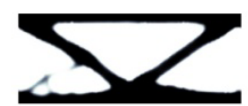

$\beta=0,2$

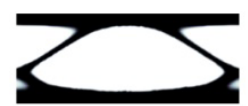

$\beta=0,5$

$$
\beta=0,8
$$

Figure 10: Iterations 0 and 30 of the unit cell initialized with the centered cubic architecture and with different values of $\beta$.

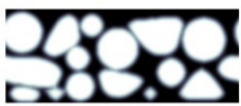

Initial shape

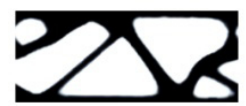

$\beta=0,2$

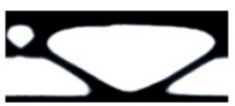

$\beta=0,5$

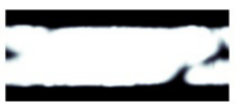

$\beta=0,8$

Figure 11: Iterations 0 and 30 of the unit cell initialized with the random architecture and with different values of $\beta$.

Looking at the evolution of the corresponding bending stiffness during optimization, fig. 12, the symmetric value of $\beta=0,5$ gives the stiffest

Centered cubic $(\mathrm{b}=0,2)$

- - - - Centered cubic $(b=0,5)$

Centered cubic $(b=0,8)$
Random $(b=0,2)$

Random $(b=0,5)$

Random $(b=0,8)$

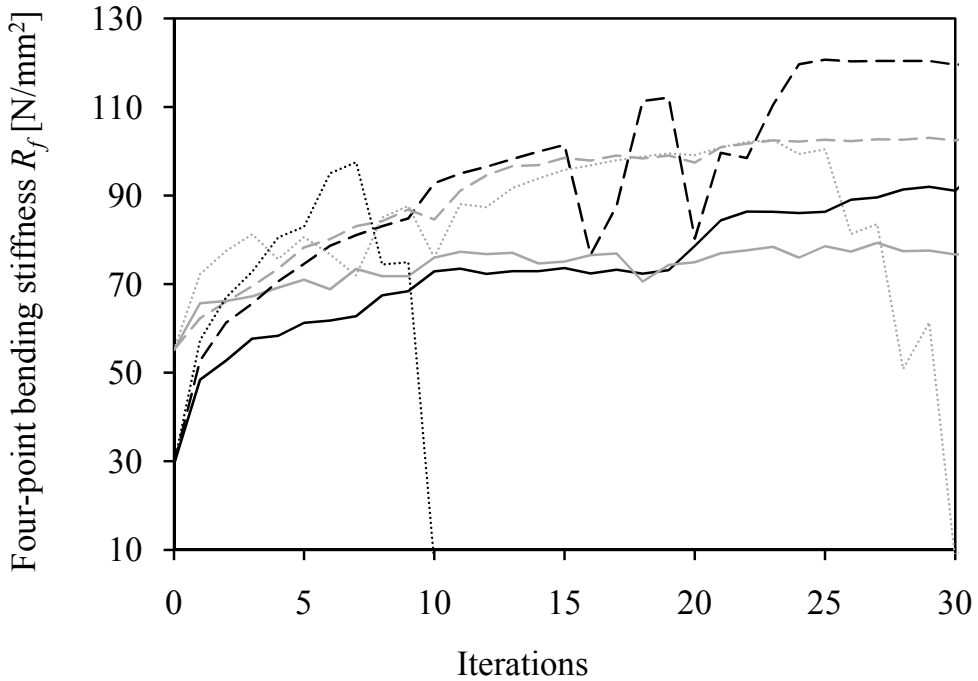

Figure 12: Evolution of the four-point stiffness $R_{f}$ (eqn. (2) with $s=50 \mathrm{~mm}$ and $\mathrm{l}=90 \mathrm{~mm}$ ) during optimization for unit cells initialized to the random architecture (black lines) and to the random architecture (grey lines). Plain lines, dashed lines and dots correspond respectively to $\beta=0.2,0.5$ and 0.8 . 
optimized architecture. Indeed, $\beta=0,2$ promotes too much the shear coefficient so that the faces are too thin. Whereas $\beta=0,8$ neglected it too much to the extent that all the matter in-between the two faces disappears. This complete disappear of matter is the result of a numerical effect at the reconditioning steps. The latter slightly modify the boundary and tend to delete the thinnest beams. All the decreases of bending stiffness observed of the figure 12 are due to this effect. Notice that quantitative comparisons between the bending performance of the final architectures are not possible because the plotted four-point bending stiffness is just an estimation as presented in Section 2.3. However, this results give interesting design directions that deserve to be considered for other functionalities and constraints (process, plastic deformation, failure mode, etc.).

\section{Conclusion}

The use of shape optimization was introduced to the conception of cellular material, like periodically architectured panels. In comparison with structural optimization, an additional homogenization procedure is needed to define relevant objective functions. In the panel case, it consists in the flexural and shear stiffness coefficients respectively computed with an applied curvature and shear strain FEM simulations on the unit cell.

Then, shape optimization was performed with objective functions as weighted sum and weighted product. The weight sum objective function leads to non admissible architectures, since one of the flexural or shear stiffness is completely neglected for the other. The weighted product gives more relevant results. For each value of the weight, different optimized architectures are obtained. Even if no quantitative comparisons were allowed, efficient architectures have been revealed and deserve to be chosen for further development steps.

An intermediate value of $\beta$ appears leading to the stiffest architectures. However, in perspective, it would be appropriated to give a physically based value of this weight, related to the specifications of the future product.

\section{References}

[1] Pollien, A., Y. Conde, L. Pambaguian and A. Mortensen. Graded opencell aluminium foam core sandwich beams. Materials Science and Engineering A, 404, 9-18, (2005).

[2] Lhuissier, P., A. Fallet, L. Salvo and Y. Brechet. Quasistatic mechanical behaviour of stainless steel hollow sphere foam: Macroscopic properties and damage mechanisms followed by X-ray tomography. Materials Letters, 63, 13-14, (2009).

[3] Queheillalt, D. T. and H. N. G. Wadley. Cellular metal lattices with hollow trusses. Acta Materialia, 53, 303-313, (2005).

[4] Wadley, H. N. G., N. A. Fleck and A. G. Evans. Fabrication and structural performance of periodic cellular metal sandwich structures. Composite Science and Technology, 63, 2331-2343, (2003). 
[5] Ashby, M. F. and Y. J. M. Brechet. Designing hybrid materials. Acta Materialia, 51, 5801-5821, (2003).

[6] Ashby, M. F., A. G. Evans, N. A. Fleck, L. J. Gibson, J. W. Hutchinson and H. N. G. Wadley. Metal Foams: A Design Guide, 2000. ButterworthHeinemann, 2000.

[7] Allen, H. G. Measurement of shear stiffness of sandwich beams. Trans. J. Plastics Inst., (1967).

[8] Buannic, N., P. Cartraud and T. Quesnel. Homogenization of corrugated core sandwich panels. Composite structures, 59, 299-312, (2003).

[9] Eschenauer, H. A. and N. Olhoff. Topology optimization of continuum structures: a review. Applied Mechanics Reviews, 54, 331-390, (2001).

[10] Allaire, G., F. Jouve and A. M. Toader. Structural optimization using sensitivity analysis and a level-set method. Journal of Computational Physics, 194, 363-393, (2004).

[11] Cea, J., S. Garreau, P. Guillaume and M. Masmoudi. The shape and topological optimizations connection. Computer Methods in Applied Mechanics and Engineering, 188, 713-726, (2000).

[12] Sethian, J. A. Level Set Methods and Fast Marching Methods: Evolving Interfaces in Computational Geometry, Fluid Mechanics, Computer Vision, and Materials Science, 1999. Cambridge University Press, 1999.

[13] Ashby, M. F. Multi-objective optimization in material design and selection. Acta Materialia, 48, 359-369, (2000). 